\title{
An Investigation of Dielectric Properties of Chicken Feather Reinforced Epoxy Matrix Composite
}

\author{
S. C. Mishra* AND NADIYA BIHARY NAYAK \\ Metallurgical and Materials Engineering Department, National Institute of \\ Technology, Rourkela, Orissa, India
}

\begin{abstract}
The present investigation aims at developing a reinforced polymer composite to be used in electronic applications (for/as high-speed data transforming insulator material). Poultry feather reinforced composite is prepared varying the reinforcement of chicken feather fibrils (i.e., with $10 \%, 20 \%$, and $30 \%$ weight fraction) using epoxy resin as matrix material. The dielectric properties of the composites are evaluated at different temperature and frequency ranges. It is found that the dielectric properties are dependent on operating frequency and temperature conditions. Such composites have potential use as a low dielectric material for typical applications. Flexural strength, microhardness, and density of this chicken feather composite are also evaluated. Microscopic observation of the feather is made with scanning electron microscope.
\end{abstract}

KEY WORDS: polymer composite, feather reinforced composite, biowaste, polymer dielectrics.

\section{INTRODUCTION}

C COMPOSITE MATERIALS ARE increasingly used for dielectric applications, i.e., applications that make use of electrically insulating or nearly insulating behavior. This is because of the need of the electronic industry for dielectric materials in electrical insulation, encapsulation, substrates, interlayer dielectrics in a multilayer ceramic chip carrier, printed circuit boards, and capacitors, and because of the rising importance of smart structures that use dielectric materials for piezoelectric, ferroelectric, and pyroelectric devices that provide sensing, actuation, etc. Chicken feather (CF) is an inconvenient and troublesome waste product of the poultry industry. The feather basically contains keratin that has ordered $\alpha$ helix or $\beta$ helix structure. Feather fiber/fibrils with an alpha helix structure at the molecular level are light and tough enough to withstand both mechanical and thermal stress [1]. Due to the hollow structure of the fiber, a given volume of fiber innately contains a significant volume of air resulting in low density, $0.80 \mathrm{~g} / \mathrm{cm}^{3}$, and low dielectric constant [2]. These fibrils have an aspect ratio $>1000$. The nodes and hooks in the hollow structure improve the structural properties and increase the surface area. Low strength of such composites restricts their use for structural applications [3-7].

*Author to whom correspondence should be addressed. E-mail: subash.mishra@gmail.com

Journal of Reinforced Plastics And Composites, Vol. 0, No. 00/2010 
However, less attention has been paid to obtaining information on thermal and electric properties of such composites. The low dielectric constant of the insulator used as printed circuit board increases the operating speed, minimizes the cross-talk effects between metal interconnects, and also diminishes the power consumption [8]. Development of low $\mathrm{k}$-dielectric material is considered to be one of the main issues in modern high-speed microelectronics. Developing a low dielectric material from locally available resources, such as $\mathrm{CF}$ fiber, is quite attractive from an economic and environmental point of view. Due to hollow structure of the fiber, a given volume of the fiber innately contains a significant volume of cavities. It is known that air is an ideal dielectric material, having a minimum dielectric constant of $\sim 1.0$ for which signals can travel faster. Air, for instance, allows the fastest movement of all because it provides essentially no resistance. When traveling near solids, however, the movement tends to kick up opposing positive charges and charges can distract the signal from completing its appointed rounds. In the present study, we have developed a new material using CF fiber as reinforcement in epoxy resin, which possesses low dielectric constant, and hence can be useful/suited for electronic applications.

\section{EXPERIMENTAL}

Epoxy LY 556, chemically belonging to the 'epoxide' family, is used as the matrix material. Its common name is bisphenol A diglycidyl ether. The hardener with IUPAC name NN0-bis (2-aminoethylethane-1,2-diamin) used with the epoxy has the designation HY-951. The epoxy resin and the hardener were supplied by Ciba Geigy India Ltd. The CFs were collected from a farmhouse at Rourkela, located in the eastern part of India. The CFs were cleaned with a polar solvent, like ethanol, and dried. The quills were removed and short fibers $(10-15 \mathrm{~mm}$ length, aspect ratio of $\sim 3000)$ were obtained. The feathers are mixed with the epoxy by stirring at room temperature till the polymerization reaction starts and they are poured in suitable molds to obtain disc-shaped samples of $12 \mathrm{~mm}$ diameter and $2.5 \mathrm{~mm}$ thickness. Four samples, sample A (pure epoxy resin), sample B (epoxy $+10 \%$ CF fiber), sample C (epoxy $+20 \% \mathrm{CF}$ fiber), and sample D (epoxy $+30 \% \mathrm{CF}$ fiber), are prepared under the same conditions of temperature and pressure.

Microhardness measurement is done using a Leitz microhardness tester. A diamond indenter, in the form of a right pyramid with a square base and an angle 1368 between opposite faces, is forced into the material under a load $F$. The two diagonals $X$ and $Y$ of the indentation left on the surface of the material after removal of the load are measured and their arithmetic mean $L$ is calculated. In the present study, the load considered $F=24.54 \mathrm{~N}$ and Vickers hardness number is calculated using the following equation:

$$
\mathrm{HV}=0: 1889 F / L^{2}
$$

and $L=X+Y / 2$, where $F$ is the applied load $(\mathrm{N}), L$ is the diagonal of square impression $(\mathrm{mm}), X$ is the horizontal length $(\mathrm{mm})$, and $Y$ is the vertical length ( $\mathrm{mm})$.

Short beam shear (SBS) tests are performed on the composite samples at room temperature to evaluate the value of flexural strength (FS). It is a three-point bend test, which generally promotes failure by interlaminar shear. The SBS test is conducted as per ASTM standard (D2344-84) using the same UTM. Span length of $40 \mathrm{~mm}$ and cross-head 
speed of $1 \mathrm{~mm} / \mathrm{min}$ are maintained. The FS of any composite specimen is determined using the following equation:

$$
\mathrm{FS}=3 P L / 2 b t^{4}
$$

where $L$ is the span length of the sample, $P$ is the load applied, and $b$ and $t$ are the width and thickness of the specimen, respectively.

The samples are coated with graphite paint on the opposite faces and heated for $5 \mathrm{~min}$ (at $1000^{\circ} \mathrm{C}$ ) in an oven for drying. Dielectric measurements are carried out at a frequency of $1 \mathrm{~Hz}$ to $1 \mathrm{MHz}$ using HP-4192A LF Impedance Analyzer, connected with a data acquisition system. The temperature is controlled with a programmable oven. All the dielectric data are collected at an interval of $5^{\circ} \mathrm{C}$, while heating at a rate of $5^{\circ} \mathrm{C} / \mathrm{min}$ at a frequency of $100 \mathrm{~Hz}$. In dielectric analysis, each sample is placed between two gold electrodes (parallel plate sensors, TA instruments). Morphology of CF fiber is examined by scanning electron microscope (SEM, JEOLJXA-840).

\section{RESULTS AND DISCUSSION}

The CF fibers are light and hollow in structure as revealed in Figure 1. It is evident from Figure 1 that the nodes and hooks in the feather bear hollow structure, which contains a significant volume of air and can impart low density as well as with good dielectric behavior. The density of CF is about $0.80 \mathrm{~g} / \mathrm{cm}^{3}$ and that of epoxy resin is about $1.1 \mathrm{~g} / \mathrm{cm}^{3}$. The density of the new dielectric materials decreases with an increase of CF content and can be made to be less than $1 \mathrm{~g} / \mathrm{cm}^{3}$ when containing the CF weight fraction of about $30 \%$.

The measured microhardness values of the different weight percentages of $\mathrm{CF}$ reinforced epoxy matrix composite are presented in Figure 2. The microhardness test result shows up to a certain limit (i.e., up to $20 \mathrm{wt} \%$ of $\mathrm{CF}$ ) hardness decreases, and after that the hardness of the composite is not much affected with a further increase in volume of feather fiber.

Figure 3 shows the comparison of FSs of the composites obtained experimentally from the three-point bend tests. There is an increase in FS with an increase in wt $\%$ of CF in
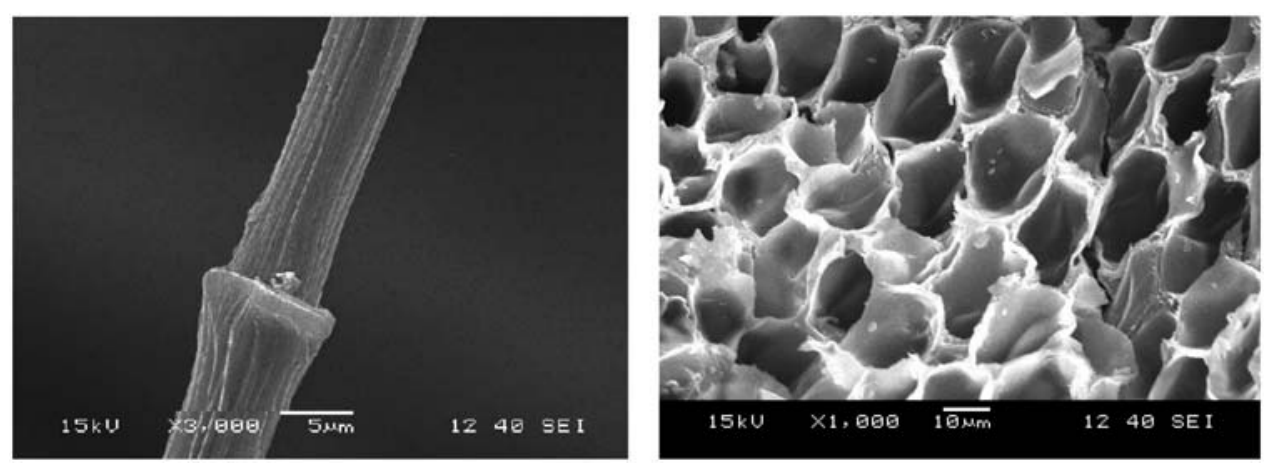

Figure 1. SEM analysis of CF fiber and its hollow structure; (a) the feather strand and (b) the cross section view of the strand. 


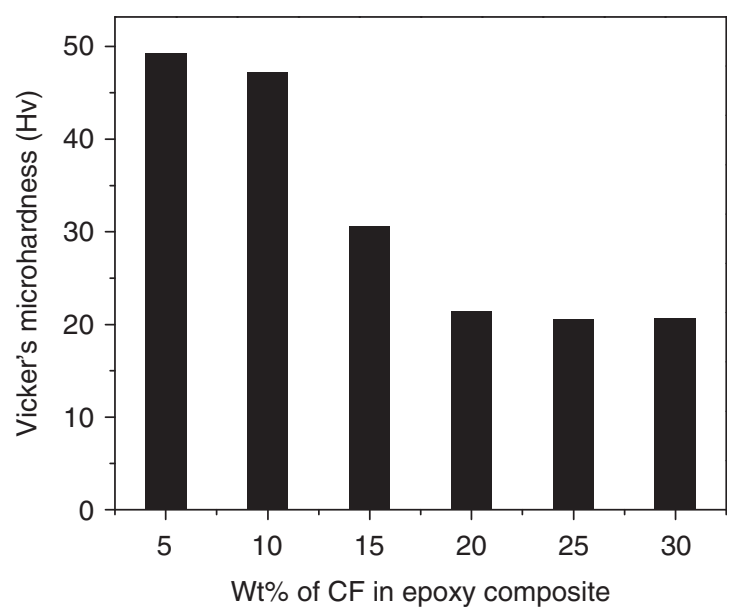

Figure 2. Variation of hardness of composites with wt\% of CF fiber.

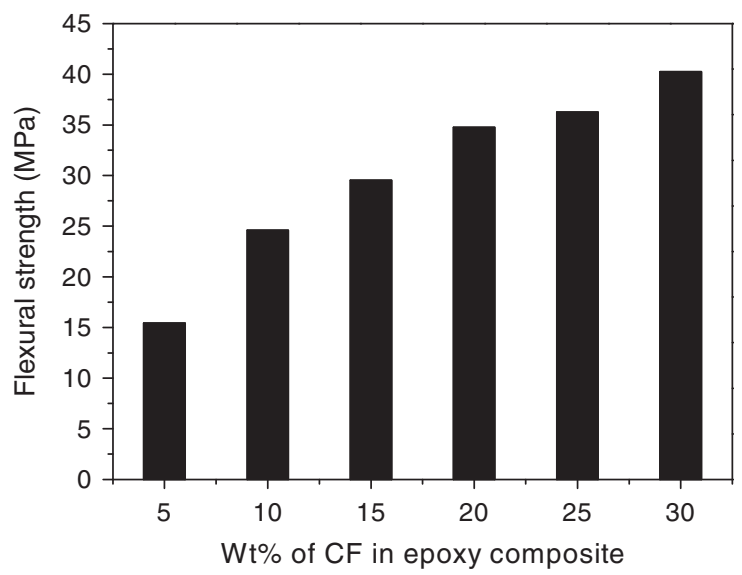

Figure 3. Variation of FS of composites with wt\% of CF fiber.

epoxy composite. This may be due to the fact that the stiffness of the fiber helps in increasing the FS of the composite.

Figure 4 shows the $k$-values of the new composite materials developed from CF fibers and epoxy resin. The $k$-values decrease from 4.5 to 2.1 , with an increase in CF content. Hence, the new CF composite has a lower dielectric constant than some conventional semiconductor insulators, epoxies, poly imides, and other dielectric materials [9]. A decrease of $k$-value of the insulator increases the operating speed. The delay time of the electronic signal is proportional to the square root of $k$, and values close to $k=1$ are most desirable [10]. The measured $k$-value of the CF itself was 1.7, which may be because $\mathrm{CF}$ fibers contain a significant volume of air. The ideal minimum $k$-value is 1.0 , as represented by air and, therefore, a porous or high-air content material may have dielectric constants in the ultra-low- $k(<2.2)$ region. 


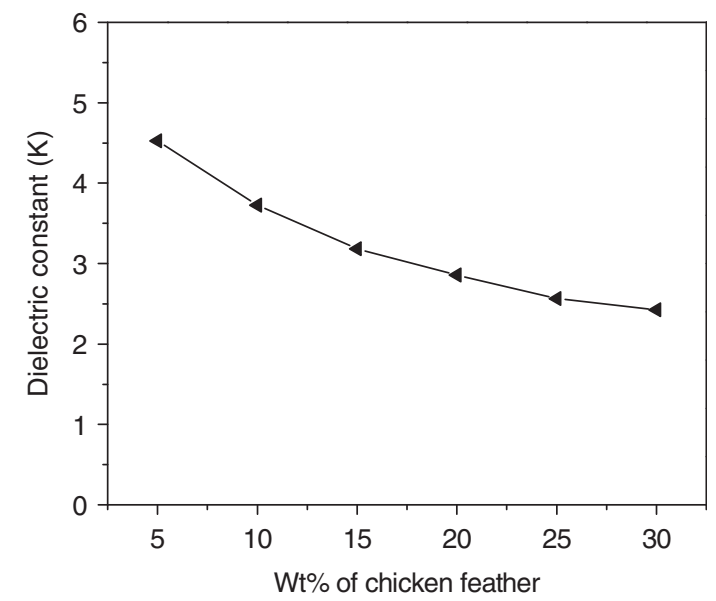

Figure 4. The dielectric constants of CF epoxy composites at $25^{\circ} \mathrm{C}$.

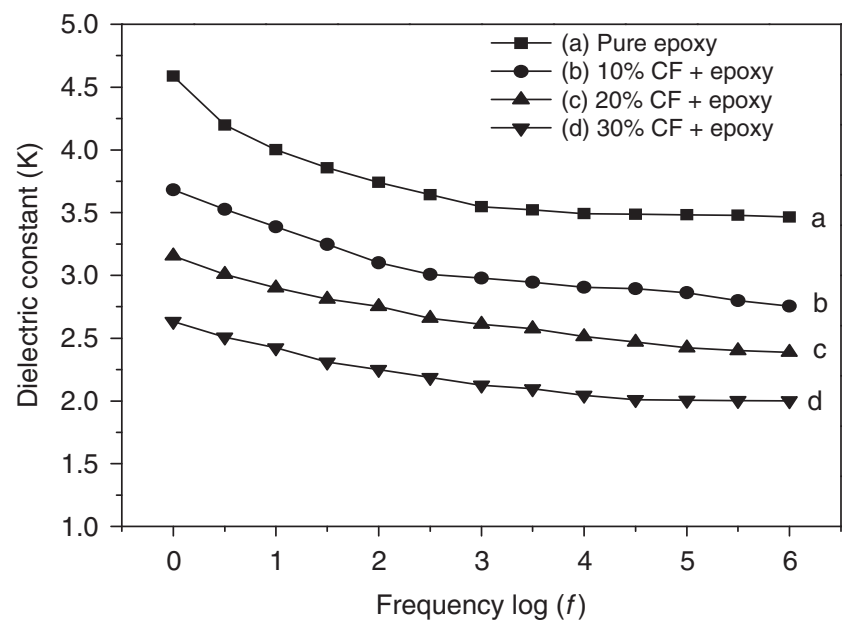

Figure 5. Frequency dependence of dielectric constant at room temperature.

The variation of the dielectric constant $k$ with frequency is shown in Figure 5. At room temperature, a marked difference in dielectric constant $k$ is found between epoxy resin and the composites prepared with different weight percentages of feather additions. An important observation is that $k$ decreases considerably with the addition of $\mathrm{CF}$ in epoxy resin, which is most likely due to the CF having a lower dielectric constant $k$ than the base epoxy resin, thus resulting in lowering the dielectric constant of these composites. The decrease of $k$ with increasing frequency is the expected behavior in most dielectric materials, which is due to dielectric relaxation and is the cause of anomalous dispersion [11]. From a structural point of view, the dielectric relaxation involves the orientation polarization, which, in turn, depends upon the molecular arrangement of the dielectric material. So, at higher frequencies the rotational motion of the polar molecules of dielectric is not sufficiently rapid for the attainment of equilibrium with the applied field, hence dielectric constant seems to decrease with increasing frequency [12]. 


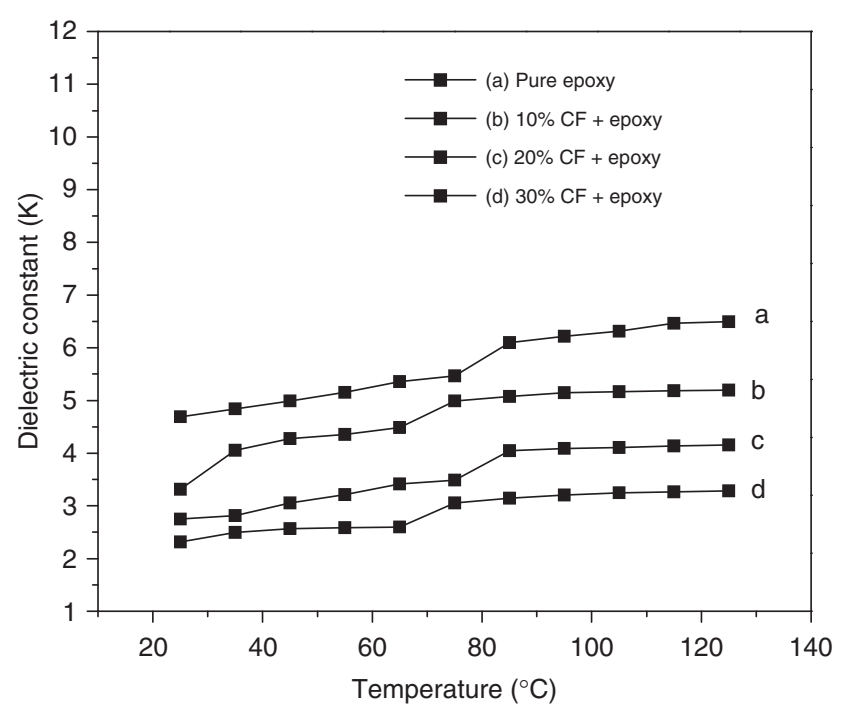

Figure 6. Temperature dependence of dielectric constant.

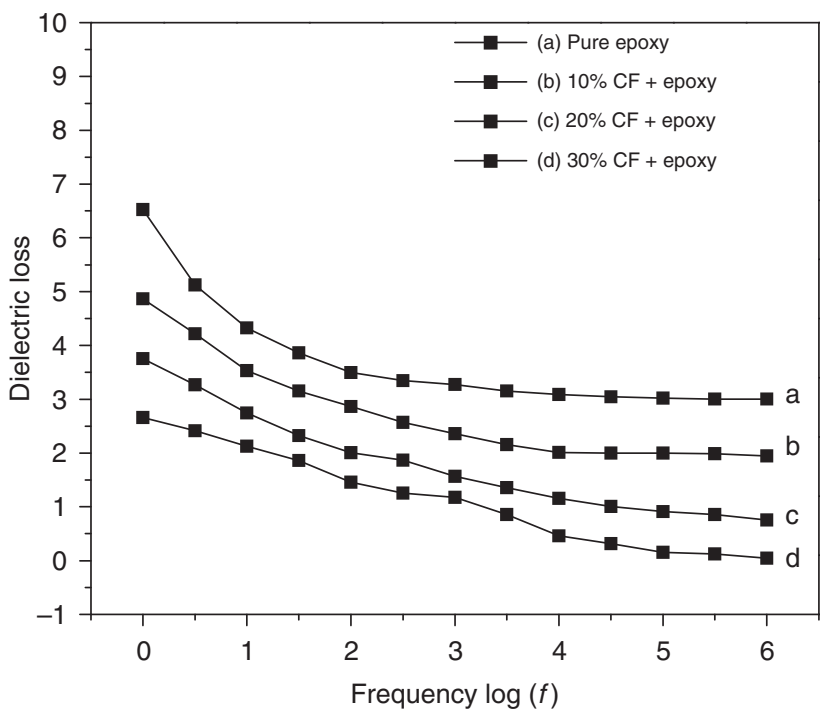

Figure 7. Frequency dependence of dielectric loss at room temperatures.

The temperature dependence of the dielectric constant of CF composites is shown in Figure 6. The dielectric constant of the composite increased slightly with increasing temperature, possibly resulting from the alignment of the dipoles when the composite softened with temperature [13].

In dielectric tests, the measured value is separated into dielectric constant and dielectric loss factor. The dependence of the loss factor upon CF content is shown in Figure 7. Loss factor represents the energy required to align the dipoles and movement of ions. In our material it is observed that the loss factor decreases with increasing CF content, which appears to be a beneficial dielectric behavior. 


\section{CONCLUSIONS}

A new low- $k$ material is developed from renewable resources using CF. The new low- $k$ composite is a natural, bio-based and environmentally friendly material. The $k$-value is found to be in the range of 4.5-1.7 depending on the CF weight fraction and temperature conditions. The $k$-values are lower than those of a conventional semiconductor insulator material such as silicon dioxide, epoxies, poly imides, and other dielectric materials, and this composite is also light in weight and rigid.

\section{ACKNOWLEDGMENTS}

This research work is partly supported by the Department of Science and Technology, Government of India, vide sanction No. 100/IFD/4246/2005-2006 dated 07.03.2006.

\section{REFERENCES}

1. Yu, M., Wu, P., Widelitz, R. B. and Chuong, C. (2002). The Morphogenesis of Feathers, Nature, 420: 308-312.

2. Kock, J.W. (2006). Physical and Mechanical Properties of Chicken Feather Materials, MS Thesis, School of Civil and Environmental Engineering, Georgia Institute of Technology.

3. Tjong, S. C. and Xu, S. A. (2004). Mechanical Properties of Glass Fiber and Liquid Crystalline Polymer Reinforced Polypropylene Hybrid Composites Toughened with Elastomers, J. Appl. Polym. Sci., 94: 1539-1546.

4. Selzer, R. and Friedrich, K. (1997). Mechanical Properties and Failure Behaviour of Carbon FiberReinforced Polymer Composites under the Influence of Moisture, Composites Part A, 28: 595-604.

5. Golden, J. H., Hawker, C. J. and Ho, P. S. (2001). Designing Porous Low-k Dielectrics, Semicond. Int., 24(5): 79-88.

6. Aoi,N. (1997), Novel Porous Films Having Low Dielectric Constants Synthesized by Liquid Phase Silylation of Spin-on Glass Sol for Intermetal Dielectrics, Japanese Journal of Applied Physics, Part 1, 36: 1355-1359.

7. Wu, W.-L., W. E. Wallace, E. K. Lin, G. W. Lynn, C. J. Glinka, E. T. Ryan, and H.-M. Ho, (2000), Properties of Nonporous Silica Thin Films Determined by High-resolution X-ray Reflectivity and Small Angle Neutron Scattering, J. Appl. Phys., 87(3) 1193-1200.

8. Martin, S. J., Godschalx, J. P., Mills, M. E., Shaffer II, E. O. and Townsend, P. (2000). Development of a Low-dielectric-constant Polymer for the Fabrication of Integrated Circuit Interconnects, Adv. Mater., 12: 1769-1778.

9. Miller, R. D. (Oct 15, 1999). In Search of Low-k Dielectrics, Science, 286(5439): 421-423.

10. Ogura, I.(1999). Low Dielectric Constant Epoxy Resins, In: Nalwa, H. S. (ed.), Handbook of Low and High Dielectric Constant Materials and their Applications, Vol.1, Chap.5, pp. 213-240, Academic Press, San Diego.

11. Daniels, C. A. (1989). Polymers - Structure and Properties, p. 32, CRC Press, Boca Raton.

12. Smyth, C. P. (1955). Dielectric Behavior and Structure, p. 53, McGraw Hill, New York.

13. Frohlick, H. (1956). Theory of Dielectrics, p. 13, Oxford University Press, Oxford. 